\title{
Examining the basis for illusory recollection: The role of remember/know instructions
}

\author{
LISA GERACI \\ Texas A\&M University, College Station, Texas \\ and \\ DAVID P. MCCABE \\ Colorado State University, Fort Collins, Colorado
}

\begin{abstract}
Curiously, studies using the remember/know paradigm to measure recollective experience show that people often vividly remember events that never occurred, a phenomenon referred to as illusory recollection. Two experiments tested the hypothesis that false remember responses in the converging associates, or Deese/Roediger-McDermott (DRM) paradigm, reflect accurate memory for the study episode, rather than false recollection of critical lures. To test this hypothesis, we used standard remember instructions that emphasized recollection of the study context by allowing participants to use memory of surrounding list items as evidence for recollection, or we used modified instructions that did not include memory for surrounding list items as a basis for recollection. Results showed that, as compared with the standard instruction condition, the modified instructions selectively reduced reports of false remember responses to critical lures, but did not affect remember responses to studied items. By contrast, remember responses to critical lures were unaffected by an instruction condition that excluded the use of voice information as evidence for remembering. These results suggest that remember responses to falsely recognized items are driven partly by retrieval of studied items, rather than illusory recollection of the critical lures themselves. They further point to the importance of instructions in influencing subjective reports.
\end{abstract}

The remember/know paradigm for studying recollective experience requires participants to use remember or know judgments to characterize the quality of their memories for items that they recognize as having studied. Participants assign remember judgments when they recognize an item and can vividly recall evidence of having encountered it in the study episode; they can remember some specific contextual detail (e.g., what they were thinking or the item's position in the study list). They assign know judgments when they recognize an item with confidence, but cannot recollect details of the item's actual occurrence; they cannot remember any details associated with the item's presentation at study (see Rajaram, 1993, for published instructions).

These two types of subjective experience were introduced as conscious states associated with output from different memory systems: Remembering was associated with retrieval from the episodic memory system, and knowing was associated with retrieval from the semantic memory system (Tulving, 1985). More recently, these states have been hypothesized to arise from different underlying retrieval processes. According to one view, these

We thank Elana Graber for her help in conducting the experiments. This research was supported by NIA Grant P50 AG056811 to L.G. Correspondence should be sent to L. Geraci, Department of Psychology, Texas A\&M University, College Station, TX 77843-4235 (e-mail: lgeraci@tamu.edu). subjective experiences reflect two independent memory processes, termed recollection and familiarity (Jacoby, 1991). Based on a dual-process model of recognition memory (Mandler, 1980), recognition can be driven by two different processes: Participants can recognize an item on a recognition memory test because they recollect having studied the item, and/or they can recognize an item because it feels familiar to them. Applied to the remember/ know paradigm, the dual-process model suggests that the recollection process gives rise to a remember experience, whereas a familiarity process gives rise to a know experience (Kelley \& Jacoby, 1998).

A complementary approach characterizes remembering and knowing in terms of the type of information that gives rise to the processes associated with these exclusive subjective experiences (Gardiner, 1988; Rajaram, 1993). In general, this research indicates that the factors that selectively or primarily increase reports of remembering are also the factors that improve free recall and recognition performance (e.g., semantic processing and generation both selectively increase reports of remembering and not knowing). According to the distinctiveness/fluency hypothesis, remember responses are affected by the distinctiveness of the study episode (Rajaram, 1996), whereas know responses are affected by the fluency of processing (Rajaram, 1993; Rajaram \& Geraci, 2000). For example, the orthographic distinctiveness effect (superior memory for unusual words, such as subpoena; see, e.g., Hunt \& 
Elliot, 1980) selectively affects remember responses but not know responses (Rajaram, 1998).

Despite the fact that remembering is usually associated with factors that increase accurate memory, recent work shows that remembering can also accompany inaccurate memories. This work shows that people often report that they vividly remember events that never occurred. In their original study, Roediger and McDermott (1995) adapted a paradigm (Deese, 1959) in which participants study words such as bed, rest, awake, and so on and falsely recognize the nonstudied (but semantically associated) word sleep. They found levels of false recognition that paralleled levels of accurate recognition, and participants claimed to vividly remember these falsely recognized items - a finding that has been termed illusory recollection (Gallo \& Roediger, 2003). Other studies have obtained evidence for illusory recollection using this paradigm (Gallo, McDermott, Percer, \& Roediger, 2001; Gallo \& Roediger, 2003; Neuschatz, Payne, Lampinen, \& Toglia, 2001; Roediger \& McDermott, 1995). In addition, illusory recollection is obtained using other false memory paradigms (Lampinen, Copeland, \& Neuschatz, 2001; Miller \& Gazzaniga, 1998).

If remembering measures vivid recollection, and is considered to reflect recollection processes involved in accurate recognition memory, then how can one understand high levels of false remembering? A reconsideration of Tulving's (1985) original conception of remembering, from which the remember/know paradigm was derived, may provide some answers to this question. In his original proposal, Tulving defined remembering as the subjective experience associated with retrieval from the episodic memory system. From this view, remembering refers to mentally reliving an episode in one's past. This conception of remembering fits well with our real-life memories of episodes. We remember having dinner last night. That is, we can put ourselves back in time and place and mentally reexperience details from the evening. This is a rich episode that is contextually defined by time and place and surrounding events, and thus it is easy to see how one can recollect this event.

How do participants remember a single word in a memory experiment? Typically, they remember that they encountered a word because they can recall perceptual details of the word - how it looked on the screen or how it sounded when spoken by the experimenter. Or, they remember the word because they can recall that the word came before or after another word in the list. Perhaps a participant tried to remember the words and formed a mnemonic (e.g., for the words girl and balloon, the participant might have formed the sentence The girl flew the balloon).

The fact that remembering reflects, in part, memory for episodic context may provide a partial explanation for the high levels of false remembering, or illusory recollection, reported in the literature using the Deese/RoedigerMcDermott (DRM) paradigm. We hypothesize that participants may claim to remember the critical lure because they recollect the episodic context provided by the items presented in the study list, and not necessarily because they vividly remember the critical lure. To test this hypoth- esis, we compared remember responses for veridical and false recognition using standard remember/know instructions (based on Rajaram, 1993) or modified instructions that did not contain the sentence instructing participants to use memory for the words that came before or after the test word as a basis for illusory recollection (see Appendix). If memory for surrounding context drives illusory recollection, then using modified instructions that do not include this information as a basis for remembering should decrease remember responses for critical lures, but have comparatively little effect on remember responses for studied items. We tested this hypothesis in two experiments.

\section{EXPERIMENT 1}

\section{Method}

Participants. Sixty-eight Washington University undergraduates participated for course credit. Half of these participants were given standard remember/know instructions, and the other half were given modified no-context instructions.

Design and Materials. The experiment was a $2 \times 4$ mixed factorial design. Instruction condition (standard vs. modified no-context) was the between-subjects variable and item type (studied target, critical lure, control target, and control lure) was the within-subjects variable. Remember/know judgments for these item types served as the dependent variable of interest.

Twenty-four 12-item lists derived from Stadler, Roediger, and McDermott's (1999) norms that have been shown to produce high levels of false recognition $(M=.70)$ were used in the experiment. These were the first 12 of the 15 words in each list, presented in the list from highest to lowest forward associative strength. Two sets of 12 lists (Set A and Set B), equated on their mean probability of false recognition in the norms, were created for counterbalancing. One set was presented at study; the other set was not presented at study and provided the control items for the recognition test.

There were 96 words on the recognition test. Three studied targets were included from each of the 12 studied lists (from list positions 1,8 , and 10 ), as well as the critical lure associated with the list. The analogous items from the 12 nonstudied lists were included as well, and served as control targets and control lures, respectively. All of these test words were presented in a random order.

Procedure. At study, the participants heard Set A or Set B presented by audiotape at a rate of 1 word every $3 \mathrm{sec}$ and were told to try to remember the words for a later memory test. The study list consisted of 12 DRM lists that were presented consecutively, with no separation between lists.

Immediately after study, the participants were given extensive remember/know/guess instructions for the recognition test. We included a guess response option, to be consistent with other recent studies of illusory recollection (e.g., Gallo et al., 2001). Half of the participants were given standard remember/know instructions, based on Rajaram's (1993) published instructions. Our version of these instructions was modified slightly to correspond to auditory study presentation (see Appendix) and to include a guess response category. The other participants were given modified instructions that included only one difference: The sentence You may even remember the words that came before or after it was removed. Thus, the explanation for what constitutes a remember response did not include memory for surrounding items (i.e., the episodic context). At the conclusion of the instructions, all of the participants were asked to provide examples of remembering, knowing, and guessing. Following the instructions, the test was administered. Test items were presented visually, one at a time, on the computer screen. The test was self-paced and the participants were told to first indicate (yes or no) whether they had heard the word earlier. If they indicated that they did recognize the item from the study list, the screen advanced 
and presented the following response options: remember, know, or guess. These response options were displayed on the screen until the participant responded, at which point the screen advanced to the next test word.

\section{Results}

The mean proportions of remember, know, and guess responses for all four item types (studied, critical lure, control lure, and control target items) are presented in Table 1. An alpha level of .05 was used for all statistical analyses. We first examined the effect of instruction condition (standard or modified) on the overall proportion of hits for studied items and false alarms to critical lures (the sum of remember, know, and guess responses for each item type). Standard instructions were associated with a marginally significant increase in hits $(M=.75)$ as compared with the modified instruction $(M=.68)$ condition $\left[F(1,66)=3.11, M S_{\mathrm{e}}=0.04, p=.08\right]$, but instructions did significantly affect the overall level of false alarms to critical lures $[M=.75$ and $M=.70$, respectively; $\left.F(1,66)=1.25, M S_{\mathrm{e}}=0.04\right]$.

We were primarily interested in the effect of the modified no-context instructions on remember responses to critical lures. We predicted that the modified instructions would selectively reduce remember responses for critical lures, but would not affect remember responses for studied items. A 2 (type of instructions: standard or modified) $\times$ 2 (item type: studied item or critical lure) ANOVA on remember responses revealed a main effect of instructions $\left[F(1,66)=9.80, M S_{\mathrm{e}}=0.44\right]$, a main effect of item type $\left[F(1,66)=7.58, M S_{\mathrm{e}}=0.18\right]$, and no significant interaction between the two variables $\left[F(1,66)=1.35, M S_{\mathrm{e}}=\right.$ $0.03]$. The lack of a significant interaction for the remember responses is somewhat difficult to interpret, given the fact that the overall hit rate was higher in the standard instruction condition than in the modified instruction condition. Because the hit rate was higher for participants in the standard instruction condition than in the modified instruction condition, the following analyses were conducted using the proportion of remember responses out of the overall number of items called "old" (cf. Rajaram, 1993). This analysis revealed a main effect of instructions $\left[F(1,66)=9.16, M S_{\mathrm{e}}=0.06\right]$, a main effect of item type $\left[F(1,66)=13.30, M S_{\mathrm{e}}=0.03\right]$, and a significant interac- tion between the two variables $\left[F(1,66)=4.15, M S_{\mathrm{e}}=\right.$ $0.03]$. The interaction between type of instructions and item type indicates that the modified instructions reduced remember responses to critical lures, whereas it had little effect on remember responses to studied items. This finding provides some support for the hypothesis that false remember responses in the standard instruction condition are partially driven by memory for surrounding study list items.

We turn now to the effect of instruction condition on remember responses for control targets and control distractors. The $2 \times 2$ ANOVA showed a main effect of item type $\left[F(1,66)=49.74, M S_{\mathrm{e}}=0.68\right]$ and instruction condition $\left[F(1,66)=93.76, M S_{\mathrm{e}}=1.36\right]$, but no interaction between the two variables $\left[F(1,66)=2.22, M S_{\mathrm{e}}=0.003\right]$. Thus, instructions did not differentially affect responding to distractor items. The proportional analysis showed the same results: a main effect of item type $[F(1,65)=34.58$, $\left.M S_{\mathrm{e}}=1.76\right]$ and instruction condition $[F(1,65)=5.43$, $\left.M S_{\mathrm{e}}=0.33\right]$, but no interaction between the two variables $\left[F(1,65)=0.38, M S_{\mathrm{e}}=0.02\right]$.

We did not have specific predictions regarding the effect of instructions on the proportion of know or guess responses, but for completeness, we report the raw data for the most relevant of these analyses. A 2 (instruction group) $\times 2$ (item type) ANOVA on the proportion of know responses revealed a main effect of item type $[F(1,66)=$ $\left.13.58, M S_{\mathrm{e}}=0.20\right]$, such that the proportion of know responses was greater for critical lures than for studied targets, but there was no main effect of instruction group and no interaction between the two variables $(p s>.10)$. Type of instructions did not differentially affect the proportion of guess responses given to studied items and critical lures (all $p \mathrm{~s}>.10$ ). The increase in know responses to critical lures in the modified group, although not statistically significant, is consistent with our hypothesis, suggesting a shift in responding such that critical lures are less likely to be associated with remembering than are studied items when list context is not included as a basis for recollection.

\section{EXPERIMENT 2}

The most intriguing result to emerge from Experiment 1 was that using remember instructions that did not direct participants to rely on memory for surrounding study

Table 1

Mean Proportions of Remember (R), Know (K), and Guess (G) Responses for Each Item Type in Experiment 1

\begin{tabular}{|c|c|c|c|c|c|c|c|c|c|c|c|c|}
\hline \multirow[b]{4}{*}{ Item Type } & \multicolumn{12}{|c|}{ Type of Instructions } \\
\hline & \multicolumn{6}{|c|}{ Standard } & \multicolumn{6}{|c|}{ Modified No-Context } \\
\hline & \multicolumn{2}{|c|}{$\mathrm{R}$} & \multicolumn{2}{|c|}{$\mathrm{K}$} & \multicolumn{2}{|c|}{ G } & \multicolumn{2}{|c|}{$\mathrm{R}$} & \multicolumn{2}{|c|}{$\mathrm{K}$} & \multicolumn{2}{|c|}{ G } \\
\hline & $M$ & $\overline{S D}$ & $M$ & $\overline{S D}$ & $M$ & $\overline{S D}$ & $M$ & $\overline{S D}$ & $M$ & $\overline{S D}$ & $M$ & $S D$ \\
\hline Studied target & .44 & .19 & .23 & .15 & .08 & .08 & .35 & .16 & .23 & .10 & .09 & .09 \\
\hline Critical lure & .39 & .21 & .28 & .18 & .08 & .12 & .25 & .18 & .34 & .15 & .11 & .11 \\
\hline Control target & .04 & .05 & .09 & .10 & .08 & .07 & .02 & .03 & .11 & .09 & .12 & .16 \\
\hline Control distractor & .21 & .19 & .20 & .16 & .06 & .07 & .13 & .13 & .19 & .12 & .13 & .16 \\
\hline
\end{tabular}

Note-Numbers refer to the mean proportions of remember, know, and guess responses out of the total number of items. 
items as evidence of recollection reduced the proportion of false remember responses to critical lures, whereas it had little effect on remember responses to studied items. The purpose of Experiment 2 was to examine this possible effect of instructions using a more dramatic instruction manipulation. In this experiment, we attempted to highlight the difference in instructions between the two groups by giving participants more elaborate instructions that included examples of each characteristic of remembering (see Appendix). We also included a third instruction condition that was designed to isolate the specific influence of our modified instructions on false remember responses. It is possible that the modified instructions reduced the proportion of remember responses given to critical lures because these instructions simply offered participants fewer characteristics to use as evidence for remembering. To examine this possibility, we included in Experiment 2 an additional condition in which a different aspect of the remember instructions was removed: the instruction to use voice information as evidence for a remember response. If the previously reported effect of instructions on the proportion of remember responses to critical lures occurred because participants had less overall detail to use to make their remember judgments, then removing another basis for remembering (voice information) from the instructions should also reduce reports of remember responses to critical lures as compared with the standard instruction condition. If, on the other hand, the previously reported reduction in illusory recollection was driven by the specific instruction to use surrounding list items as evidence for remembering, then results from this new instruction condition (hereafter called the modified no-voice instruction condition) should not reduce illusory recollection as compared with the standard condition. Rather, the reduction in illusory recollection should be isolated to the original modified no-context instruction condition.

\section{Method}

Participants. One hundred and twenty-six Washington University undergraduates participated for course credit. One third (42) of the participants were given standard instructions, one third were given the modified no-context instructions, and one third were given the modified no-voice instructions.

Design, Materials, and Procedure. The design, materials, and procedure were identical to those used in Experiment 1, with the following exceptions. In this experiment, we used longer, more standard study lists than we did in Experiment 1. We used the same 24 lists as in Experiment 1, but we used the entire 15-item lists for each list (from Roediger, Watson, McDermott, \& Gallo, 2001), and divided the lists into three different study sets of 8 lists. Two sets of items served as studied lists and the remaining set that was not presented at study provided the control items for the recognition test. The three sets were counterbalanced across and between groups such that each was used an equal number of times (as studied items and distractors) for each instruction group. Studied words were also presented at a faster, more standard rate of presentation of $2 \mathrm{sec}$ per item.

Remember/know instructions in Experiment 2 were enhanced by giving the participants examples of each characteristic that might indicate that they were remembering. Consistent with Experiment 1, the participants were given standard instructions, with the statement regarding the use of surrounding items as evidence of remembering; modified instructions, without the surrounding item section; or modified instructions, without the voice instruction (see Appendix).

\section{Results}

For Experiment 2, the mean proportion of remember, know, and guess responses for all four types of items (studied, critical lure, control lure, and control target items) are presented in Table 2. In all of the following analyses, we examined the effect of each modified instruction condition as compared with the standard condition. Therefore, we first examined the effect of instruction type (standard vs. modified no context) on the overall proportion of hits. This analysis showed that the hit rate for studied items did not differ significantly between the standard instruction $(M=.61)$ and the modified no-context instruction $(M=.65)$ conditions $\left[F(1,82)=1.65, M S_{\mathrm{e}}=0.02\right]$. Similarly, the overall level of false alarms to critical lures was unaffected by this instruction manipulation $[M=.69$ in the standard and $M=.71$ in the modified no-context instruction condition; $\left.F(1,82)=0.14, M S_{\mathrm{e}}=0.04\right]$. We also examined hits and false alarms for the modified novoice condition as compared with the standard instruction condition. Results showed that the hit rate was unaffected by these two instruction conditions $[M=.61$ for standard and $M=.64$ for the modified no-voice condition; $\left.F(1,82)=0.97, M S_{\mathrm{e}}=0.02\right]$. In addition, false alarms to critical lures were not affected by these instructions ( $M=$ .69 in both instruction conditions).

Next, we analyzed the effect of instruction condition on reports of remembering and knowing. Because there was no difference in the hits or false alarm rates across instruction condition, the following analyses were conducted on the proportion of remember and know responses out of the total number of studied or critical items. We note, though,

Table 2

Mean Proportions of Remember (R), Know (K), and Guess (G) Responses for Each Item Type in Experiment 2

\begin{tabular}{|c|c|c|c|c|c|c|c|c|c|c|c|c|c|c|c|c|c|c|}
\hline \multirow[b]{4}{*}{ Item Type } & \multicolumn{18}{|c|}{ Type of Instructions } \\
\hline & \multicolumn{6}{|c|}{ Standard } & \multicolumn{6}{|c|}{ Modified No-Context } & \multicolumn{6}{|c|}{ Modified No-Voice } \\
\hline & \multicolumn{2}{|c|}{$\mathrm{R}$} & \multicolumn{2}{|c|}{$\mathrm{K}$} & \multicolumn{2}{|c|}{$\mathrm{G}$} & \multicolumn{2}{|c|}{$\mathrm{R}$} & \multicolumn{2}{|c|}{$\mathrm{K}$} & \multicolumn{2}{|c|}{$\mathrm{G}$} & \multicolumn{2}{|c|}{$\mathrm{R}$} & \multicolumn{2}{|c|}{$\mathrm{K}$} & \multicolumn{2}{|c|}{ G } \\
\hline & $M$ & $\overline{S D}$ & $M$ & $\overline{S D}$ & $M$ & $\overline{S D}$ & $M$ & $\overline{S D}$ & $M$ & $\overline{S D}$ & $M$ & $\overline{S D}$ & $M$ & $S D$ & $M$ & $\overline{S D}$ & $M$ & $S D$ \\
\hline Studied target & .32 & .18 & .18 & .10 & .11 & .09 & .34 & .16 & .19 & .11 & .13 & .09 & .31 & .16 & .22 & .12 & .11 & .07 \\
\hline Critical lure & .32 & .20 & .26 & .14 & .12 & .10 & .27 & .19 & .30 & .19 & .13 & .11 & .31 & .20 & .26 & .15 & .12 & .13 \\
\hline Control target & .05 & .11 & .09 & .09 & .11 & .12 & .03 & .05 & .09 & .10 & .13 & .11 & .03 & .06 & .09 & .10 & .14 & .11 \\
\hline Control distractor & .07 & .12 & .10 & .12 & .12 & .14 & .05 & .10 & .15 & .14 & .13 & .18 & .04 & .07 & .12 & .14 & .16 & .14 \\
\hline
\end{tabular}

Note-Numbers refer to the mean proportions of remember, know, and guess responses out of the total number of items. 
that the outcomes for each statistical analysis are the same when remember and know responses are examined as a proportion of the hit or false alarm rates. As in Experiment 1 , the comparison of primary interest was the effect of instructions on remember responses to critical lures. First we examined the comparison between the standard instruction condition and the modified no-context instruction condition. A 2 (type of instructions: standard or modified) $\times 2$ (item type: studied item or critical lure) ANOVA revealed no main effect of instructions $[F(1,82)=0.19$, $\left.M S_{\mathrm{e}}=0.06\right]$, a main effect of item type $[F(1,82)=4.86$, $\left.M S_{\mathrm{e}}=0.01\right]$, and a significant interaction between the type of instructions and remember responses for studied and critical items $\left[F(1,82)=4.17, M S_{\mathrm{e}}=0.01\right]$. This interaction replicates the results from the proportional analyses in Experiment 1 and shows that the modified instructions selectively reduced remember responses to critical lures, whereas they had little effect on remember responses to studied items (see Figure 1). As in Experiment 1, this same ANOVA performed on control targets and control distractors showed no main effects of item type $\left[F(1,82)=1.36, M S_{\mathrm{e}}=0.02\right]$, or instruction condition $\left[F(1,82)=3.31, M S_{\mathrm{e}}=0.004\right]$, and no interaction between the two variables $\left[F(1,82)=.02, M S_{\mathrm{e}}=0.004\right]$. Thus, instructions did not differentially affect responding to distractors.

Again, we did not have specific predictions regarding the effect of instructions on the proportion of know or guess responses, but for completeness, we report the most relevant of these analyses. A 2 (instruction group) $\times 2$ (item type) ANOVA on the proportion of know responses revealed no main effect of instructions $[F(1,82)=1.01$, $\left.M S_{\mathrm{e}}=0.03\right]$, a main effect of item type $[F(1,82)=33.30$, $\left.M S_{\mathrm{e}}=0.01\right]$, but no significant interaction $[F(1,82)=$ $\left.1.59, M S_{\mathrm{e}}=0.01\right]$. The type of instructions did not differentially affect guess responses either $(p s>.10)$. Un-

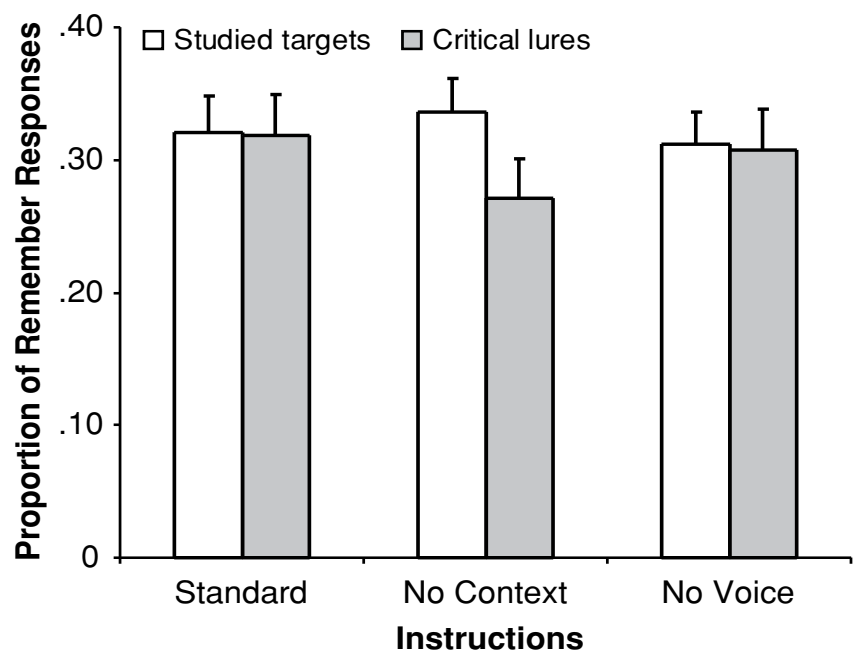

Figure 1. Proportions of remember responses out of the total number of recognized studied items given to studied items and critical lures for each instruction condition in Experiment 2. like in Experiment 1, which showed an increase in know responses for critical lures when modified instructions were given, in this experiment, know and guess responses did not interact with type of instructions. Thus, the type of instructions only affected remember responses given to critical lures in Experiment 2.

To examine whether this effect of instructions on false remember responses was driven by a lack of reliance on surrounding list items, or on a more general lack of reliance on detail, we next compared the data from the standard condition with the modified no-voice condition. If the reported reduction in remember responses in the nocontext condition is driven by a general lack of reliance on recollective details, then remember responses should be similarly reduced by the no-voice instruction condition, as compared with the standard instruction condition. If, on the other hand, the no-voice instructions do not reduce false remember responses, then we would have evidence that the previous reduction in false remembering is specifically driven by diminished reliance on memory for surrounding list items. A 2 (type of instructions: standard or no voice) $\times 2$ (item type: studied item or critical lure) ANOVA revealed that there were no main effects of instructions $\left[F(1,82)=0.09, M S_{\mathrm{e}}=0.06\right]$ or item type $\left[F(1,82)=0.05, M S_{\mathrm{e}}=0.01\right]$. Importantly for present purposes, there was also no interaction between the two variables $\left[F(1,82)=0.01, M S_{\mathrm{e}}=0.01\right]$, showing that remember responses to critical lures were not differentially affected by the modified no-voice instructions. For know responses, there was no effect of instructions $[F(1,82)=$ $\left.0.57, M S_{\mathrm{e}}=0.02\right]$, but there was a main effect of item type $\left[F(1,82)=16.65, M S_{\mathrm{e}}=0.01\right]$, showing that there were more know responses given to critical lures than to studied items overall. The interaction between the two variables was not significant $\left[F(1,82)=1.29, M S_{\mathrm{e}}=0.01\right]$.

The results from Experiment 2 demonstrate that when participants are not instructed to use memory for surrounding list items as evidence of remembering, they are less likely to assign remember responses to critical false alarms. By contrast, this instruction manipulation did not change participants' likelihood of indicating that they remembered studied items. The absence of a reduction in false remember responses in the no-voice instruction condition in conjunction with the presence of such a reduction in the no-context instruction condition indicates that false remember responses are partly driven by memory for surrounding list items. When another characteristic of remembering (i.e., recalling voice information) was eliminated from the instructions, reports of remember responses to critical lures were not differentially reduced as compared with reports of remember responses to studied items. Thus, we demonstrate that our modified instructions selectively affected remember responses to critical lures, as compared with remember responses to studied items. We conclude that a significant portion of illusory recollection in this paradigm comes from accurate episodic memory for study context, rather than retrieval of the never-presented item. 


\section{DISCUSSION}

Across two experiments with slightly different methods, our results showed that false remember responses in the DRM paradigm are influenced by accurate memory for the study context. Experiments 1 and 2 replicated previous studies showing high levels of illusory recollection when participants were given standard remember instructions that emphasized recollection of the study context and allowed memory of surrounding list items to constitute evidence of recollection. However, in both experiments, reports of illusory recollection were substantially reduced relative to accurate recollection when instructions were used that deemphasized study context, and shifted responding to more item-specific characteristics. Reports of illusory recollection were not reduced when another aspect of remembering (i.e., memory for voice), was eliminated from the instructions. These results indicate that illusory recollection is partly driven by accurate memory for study context.

How do instructions influence remember responses? A signal detection view would suggest that the modified nocontext instructions reduced remember responses to critical lures by causing participants to be more conservative in their recognition and remember judgments. The results from Experiment 1 could be taken to be consistent with this view, because, in addition to reducing the proportion of remember responses to critical lures, the modified no-context instructions also reduced overall recognition performance as compared with the standard instruction condition. Thus, one could interpret these data to mean that the instruction manipulation influenced participants' criterion placement, and not their selective use of specific information to make a remember judgment. A signal detection view is more difficult to apply to the results of Experiment 2 , in which overall recognition performance did not change across the standard and modified no-context instruction conditions, whereas remember responses decreased only for critical lures and not for studied items or control items. Thus, it seems unlikely that the effects of instructions on false remember responses can be explained by a shift in response criteria. Instead, there seems to be good evidence that the reduction in false remember responses occurred because people did not rely on memory for surrounding list items to influence their subjective judgments of recollection.

Our results suggest one mechanism that influences illusory recollection, but there are other factors contributing to illusory recollection as well. One idea is that people misattribute (Gallo \& Roediger, 2003) or incorrectly bind (Hicks \& Hancock, 2002) features from studied items to a related nonstudied item. Indeed, there is evidence for this mechanism. For example, when people falsely recognize the critical lure, they claim that it was presented in the same modality (Gallo et al., 2001) as the presented items in the list. In addition, a growing body of research on realitymonitoring errors demonstrates that people falsely believe that they perceived events that they only imagined, if these perceived events share physical, semantic (Henkel, Frank- lin, \& Johnson, 2000), and even linguistic features (Geraci \& Franklin, 2004). Thus, it is certainly possible for illusory recollection to occur because people misattribute or bind features from studied items to nonstudied items. Regardless of whether or not people imagine nonstudied items and thus experience reality-monitoring errors, our data suggest that correct episodic memory for the surrounding items is responsible for a substantial proportion of illusory recollection in the DRM paradigm. Thus, it appears that false remember responses arise partly from memory for list context, rather than from memory for item features. Indeed, some researchers have suggested that in this paradigm, participants base their responses on memory for relational or thematic information because this information is most salient (Mather, Henkel, \& Johnson, 1997) and because they have poor memory for item-specific details (McCabe, Presmanes, Robertson, \& Smith, 2004). One idea is that participants may have information available that can allow them to differentiate between veridical and false recognition, but they do not use that information unless experimental conditions encourage it (McCabe \& Smith, 2002).

Whereas the instruction manipulation reduced false remember responses in the present study, it did not completely eliminate them. Therefore, it may be that other mechanisms, such as the ones just described, also mediate the effect of illusory recollection. Alternatively, it is possible that not including certain bases for remember responses in the instructions given to participants reduces the chances that participants will use that information as a basis for assigning remember responses, but it does not eliminate the possibility. In other words, although the instruction manipulation provides evidence for the idea that false remember responses may be driven by memory for context, the manipulation may not have been powerful enough to completely prevent participants from using list context to inform their remember responses.

Nonetheless, our results do point to the importance of examining the role of remember/know instructions in various experimental paradigms. Although the importance of instructions for subjective reports of remembering has been the focus of informal discussions, it has not received much empirical study. The way in which participants interpret the remember/know distinction certainly determines what these responses reflect, and participants' interpretations of these response categories largely reflect the type of information that is available in memory (Bodner \& Lindsay, 2003). Therefore, the issue of how participants define and interpret instructions for subjective reports of remembering has both practical and theoretical implications.

\section{REFERENCES}

BoDNER, G. E., \& LiNDSAY, D. S. (2003). Remembering and knowing in context. Journal of Memory \& Language, 48, 563-580.

DEESE, J. (1959). On the prediction of occurrence of particular verbal intrusions in immediate recall. Journal of Experimental Psychology, $\mathbf{5 8}, 17-22$

Gallo, D. A., McDermott, K. B., Percer, J. M., \& Roediger, H. L., III (2001). Modality effects in false recall and false recognition. Jour- 
nal of Experimental Psychology: Learning, Memory, \& Cognition, 27, 339-353

Gallo, D. A., \& Roediger, H. L., III (2003). The effects of associations and aging on illusory recollection. Memory \& Cognition, 31, 1036-1044.

GARDINER, J. M. (1988). Functional aspects of recollective experience. Memory \& Cognition, 16, 309-313.

Geraci, L., \& FrankLIN, N. (2004). The influence of linguistic labels on source-monitoring decisions. Memory, 12, 571-585.

Henkel, L. A., \& Franklin, N., \& Johnson, M. K. (2000). Crossmodal source monitoring confusions between perceived and imagined events. Journal of Experimental Psychology: Learning, Memory, \& Cognition, 26, 321-335.

HICKS, J. L., \& HANCOCK, T. W. (2002). Backward associative strength determines source attributions given to false memories. Psychonomic Bulletin \& Review, 9, 807-815.

Hunt, R. R., \& ElLIOT, J. M. (1980). The role of nonsemantic information in memory: Orthographic distinctiveness effects on retention. Journal of Experimental Psychology: General, 109, 49-74.

JACOBY, L. L. (1991). A process dissociation framework: Separating automatic from intentional uses of memory. Journal of Memory \& Language, 30, 513-541.

KelleY, C. M., \& JACOBY, L. L. (1998). Subjective reports and process dissociation: Fluency, knowing, and feeling. Acta Psychologica, 98, 127-140.

Lampinen, J. M., Copeland, S. M., \& Neuschatz, J. S. (2001). Recollections of things schematic: Room schemas revisited. Journal of Experimental Psychology: Learning, Memory, \& Cognition, 27, 1211-1222.

MANDLER, G. (1980). Recognizing: The judgment of previous occurrence. Psychological Review, 87, 252-271.

Mather, M., Henkel, L. A., \& Johnson, M. K. (1997). Evaluating characteristics of false memories: Remember/know judgments and memory characteristics questionnaire compared. Memory \& Cognition, 25, 826-837.
McCabe, D. P., Presmanes, A. G., Robertson, C. L., \& Smith, A. D. (2004). Item-specific processing reduces false memories. Psychonomic Bulletin \& Review, 11, 1074-1079.

McCABE, D. P., \& SMith, A. D. (2002). The effect of warnings on false memories in young and older adults. Memory \& Cognition, 30, $1065-$ 1077.

Miller, M. B., \& Gazzaniga, M. S. (1998). Creating false memories for visual scenes. Neuropsychologia, 36, 513-520.

Neuschatz, J. S., Payne, D. G., Lampinen, J. M., \& Toglia, M. P. (2001). Assessing the effectiveness of warnings and the phenomenological characteristics of false memories. Memory, 9, 53-71.

RAJARAM, S. (1993). Remembering and knowing: Two means of access to the personal past. Memory \& Cognition, 21, 89-102.

Rajaram, S. (1996). Perceptual effects on remembering: Recollective processes in picture recognition memory. Journal of Experimental Psychology: Learning, Memory, \& Cognition, 22, 365-377.

RAJARAM, S. (1998). The effects of conceptual salience and perceptual distinctiveness on conscious recollection. Psychological Bulletin \& Review, 5, 71-78.

Rajaram, S., \& Geraci, L. (2000). Conceptual fluency selectively influences knowing. Journal of Experimental Psychology: Learning, Memory, \& Cognition, 26, 1070-1074.

Roediger, H. L., III, \& MCDermott, K. B. (1995). Creating false memories: Remembering words not presented in lists. Journal of Experimental Psychology: Learning, Memory, \& Cognition, 21, 803-814.

Roediger, H. L., III, Watson, J. M., McDermott, K. B., \& Gallo, D. A. (2001). Factors that determine false recall: A multiple regression analysis. Psychonomic Bulletin \& Review, 8, 385-407.

Stadler, M. A., Roediger, H. L., III, \& McDermott, K. B. (1999). Norms for word lists that create false memories. Memory \& Cognition, 27, 494-500.

Tulving, E. (1985). Memory and consciousness. Canadian Psychology, 26, 1-12. 


\section{APPENDIX}

\section{Experiment 1 Remember Instructions}

You should make a remember judgment, by pressing the $R$ key, if you can consciously recollect the word's prior occurrence. That is, you would press $R$ if you can mentally travel back in time to the specific moment that you heard the word. For example, you may recall the specific sound of the voice that spoke the word, or you may recall something that happened in the room when that word was presented (a noise), or you may recall a specific thought you had about that particular word at the moment it was presented. You may even remember the words that came before or after it. The important point is that you should indicate that you remember the word if you can become consciously aware again of some particular details of the word's occurrence. So, you should respond $R$ only if the word brings to mind particular vivid details from encountering the word during the study list.

\section{Experiment 2 Remember Instructions}

\section{No Context}

You should make a remember judgment, by pressing the $R$ key, if you can consciously recollect the word's prior occurrence. That is, you would press $R$ if you can mentally travel back in time to the specific moment that you heard the word. You may recall the specific sound of the voice that spoke the word. For example, you may remember a particular oddity in the pronunciation, or a particular lisp sound at the beginning or end of the word. Or, you may recall something that happened in the room when that word was presented. For example, you may recall hearing a noise, or someone coughing at the same instant that the word was spoken on the tape. You may recall a personal association you made about the word, such as its relation to yourself or your own life. You may also recall a specific image of the word that you formed. For example, if you visualized the word, then you might remember that image you formed.

Lastly, you may even remember the words that came before or after that word in the list. You may be able to remember a story associating these words, or remember relating them to one another in some way.

\section{No Voice}

You should make a remember judgment, by pressing the $R$ key, if you can consciously recollect the word's prior occurrence. That is, you would press $R$ if you can mentally travel back in time to the specific moment that you heard the word. You may recall the specific sound of the voice that spoke the word. For example, you may remember a particular oddity in the pronunciation, or a particular lisp sound at the beginning or end of the word. You may recall something that happened in the room when that word was presented. For example, you may recall hearing a noise, or someone coughing at the same instant that the word was spoken on the tape. You may recall a personal association you made about the word, such as its relation to yourself or your own life. You may also recall a specific image of the word that you formed. For example, if you visualized the word, then you might remember that image you formed.

Lastly, you may even remember the words that came before or after that word in the list. You may be able to remember a story associating these words, or remember relating them to one another in some way.

Note-The italicized sentence is omitted in the modified instruction conditions for both experiments. 